\title{
Factores de riesgo del bajo peso al nacer, Hospital Gineco-Obstétrico Provincial de Sancti Spíritus, Cuba
}

\author{
Marcos Aurelio Grau Espinosa, ${ }^{1}$ Luís Saenz Darias ${ }^{1}$ \\ y José Andrés Cabrales Escobar ${ }^{2}$
}

RESUMEN El objetivo de este trabajo fue identificar algunos factores de riesgo que pudiera presentar la madre durante el embarazo, asociados con la incidencia de bajo peso al nacer (menos de 2500 g). Se diseñó un estudio con 378 casos y 649 controles del Hospital Gineco-Obstétrico Provincial de Sancti Spíritus, Cuba, durante el año 1994. Los datos se obtuvieron de la histo ria clínica, el registro de partos y una entrevista personal con la madre. Se realizó un análisis bivariado y se controlaron los posibles factores de confusión utilizando la regresión logística dicotómica, mediante los programas Epi Info 5 y SPSS. Por medio del modelo multivariado final se identificaron como factores de riesgo significativos de bajo peso al nacer la hipertensión arterial durante el embarazo, la consulta extemporánea de evaluación del embarazo, los abor tos anteriores, último intervalo intergenésico menor de 2 años y el aumento de peso materno menor de $8 \mathrm{~kg}$. Se debe dar prioridad a estas tres últimas variables para mantener buenos in dicadores en el programa maternoinfantil de la Provincia de Sancti Spíritus, Cuba.

Las políticas de prevención deben tomar en cuenta los factores que influyen en la aparición de los problemas de salud. En el caso del bajo peso al nacer (BPN) se han identificado varios factores de riesgo relacionados con características de la madre y del embarazo: antecedentes de BPN (1-4), ruptura prematura de membranas (1), abortos espontáneos o inducidos $(2,5)$; hipertensión arterial durante el embarazo (3, $6,7)$; carencia de esposo o apoyo conyugal $(2,8)$; atención prenatal inadecuada

\footnotetext{
1 Hospital Pediátrico Provincial de Sancti Spíritus y Facultad de Ciencias Médicas de Sancti Spíritus, Cuba. Toda correspondencia debe dirigirse a José Andrés Cabrales Escobar a la siguiente dirección postal: Edificio 3, Apto. 19, Olivos 3 Sancti Spíritus, Cuba.

2 Facultad de Ciencias Médicas de Sancti Spíritus, Cuba.
}

(8-10); tabaquismo durante el embarazo (2, 7-12); edad de la madre menor de 17,19 ó 20 años $(3,7,12)$ o mayor de 35 ó $36(2,7)$; intervalo intergenésico menor de 12 meses $(7,13)$; bajo nivel de escolaridad (10), y poco aumento de peso durante el embarazo (12).

Así, el objetivo de este trabajo fue identificar algunos factores de riesgo que pudieran asociarse con la incidencia de bajo peso al nacer (menos de 2500 g) en el Hospital Gineco-Obstétrico Provincial (HGOP) de Sancti Spíritus, Cuba, durante 1994.

\section{MATERIAL Y MÉTODOS}

Se diseñó un estudio de casos y controles, incluyéndose como casos a todos los niños nacidos vivos de parto simple (no múltiple), con un peso menor de $2500 \mathrm{~g}$ al nacer, independientemente de la edad gestacional, entre el 1 de enero y el 31 de diciembre de 1994 en el HGOP y que residieran en la provincia de Sancti Spíritus, Cuba.

Los controles fueron niños que nacieron vivos en el mismo hospital inmediatamente antes o después de cada caso, con un peso igual o superior a $2500 \mathrm{~g}$, y que procedían de la misma provincia de Sancti Spíritus.

Las fuentes de información fueron las historias clínicas de las embarazadas, el registro de partos del hospital y una entrevista personal con la madre realizada por el médico de familia que atendió el embarazo y puerperio, entrenado previamente.

Se investigaron las siguientes variables de la madre, por considerarse po- 
sibles factores de riesgo de bajo peso al nacer: edad (menor de 17 años y mayor de 35), escolaridad (primaria incompleta o menos), estado conyugal (carencia de pareja), embarazos anteriores (tres o más), abortos anteriores, historia de hijos con bajo peso al nacer, intervalo intergenésico (menor de 24 meses), tabaquismo durante el embarazo, hipertensión arterial durante el embarazo, ruptura prematura de membranas, aumento de peso durante el embarazo menor de $8 \mathrm{~kg}$; control tardío del embarazo (después de 14 semanas), consultas extemporáneas de evaluación (15 días después del primer control) y seguimiento (entre las semanas 28 y 32) del embarazo.

Se realizó un análisis bivariado utilizando el programa Epi Info 5. Se estimó cada razón de posibilidades (odds ratio, $\mathrm{RP}$ ) con un intervalo de confianza de $95 \%$; se consideró significativo el resultado cuando el límite inferior del intervalo era mayor de uno. Para el control de los posibles factores de confusión se hizo un análisis multivariado mediante regresión logística dicotómica, utilizando el procedimiento "hacia delante" de Wald (Wald's forward step analysis). En cada paso se utilizó como criterio una $P$ de 0,05 para añadir una variable y de 0,1 para eliminarla. Este análisis multivariado se realizó con el programa SPSS, previa transferencia de la base de datos desde Epi Info y recodificación de las variables. Se asignó el valor de uno a los casos y de cero a los controles; uno a la exposición y cero a su ausencia. Para la significación estadística de la RP en el análisis multivariado se utilizó el estadístico de Wald, que distribuye ji al cuadrado $\left(\chi^{2}\right)$ con un grado de libertad, con un nivel de significación de 0,05 .

Como medida de impacto se utilizó el riesgo atribuible poblacional (RAP) porcentual, calculado a partir de la siguiente fórmula (14):

$$
\mathrm{RAP}=\frac{p(E)(R R-1)}{1+p(E)(R R-1)}
$$

En donde:

$$
p=\text { proporción de la población. }
$$

CUADRO 1. Factores de riesgo de bajo peso al nacer (BPN) en 378 casos y 649 controles.

\begin{tabular}{|c|c|c|c|c|c|c|}
\hline \multirow[b]{2}{*}{ Factor de riesgo } & \multicolumn{2}{|c|}{ Expuestos } & \multicolumn{2}{|c|}{ No expuestos } & \multirow[b]{2}{*}{$\mathrm{RP}^{\mathrm{a}}$} & \multirow[b]{2}{*}{ IC95\% de la RP } \\
\hline & Casos & Controles & Casos & Controles & & \\
\hline $\begin{array}{l}\text { Hipertensión arterial durante } \\
\text { el embarazo }\end{array}$ & 29 & 25 & 349 & 624 & 2,07 & 1,16 a 3,72 \\
\hline Ruptura prematura de membranas & 31 & 31 & 347 & 618 & 1,78 & 1,03 a 3,07 \\
\hline $\begin{array}{l}\text { Aumento de menos de } 8 \mathrm{~kg} \\
\text { durante el embarazo }\end{array}$ & 127 & 104 & 251 & 545 & 2.65 & 1,94 a 3.62 \\
\hline Antecedentes de hijos con BPN & 34 & 35 & 344 & 614 & 1,73 & 1,03 a 2,91 \\
\hline Tres o más embarazos anteriores & 64 & 76 & 314 & 573 & 1,54 & 1,06 a 2,24 \\
\hline Abortos anteriores & 170 & 244 & 208 & 405 & 1,36 & 1,04 a 2,65 \\
\hline Intervalo intergenésico $<24$ meses & 55 & 60 & 147 & 259 & 1,62 & 1,04 a 2,51 \\
\hline Tabaquismo durante el embarazo & 87 & 102 & 291 & 547 & 1,60 & 1,15 a 2,23 \\
\hline Edad de la madre $<17$ años & 23 & 42 & 339 & 589 & 0,95 & 0,54 a 1,66 \\
\hline Edad de la madre > 35 años & 16 & 18 & 339 & 589 & 1,54 & 0,74 a 3,22 \\
\hline $\begin{array}{l}\text { Primer control tardío del embarazo } \\
\text { (después de } 14 \text { semanas) }\end{array}$ & 72 & 83 & 306 & 566 & 1,60 & 1,12 a 2,30 \\
\hline $\begin{array}{l}\text { Consulta extemporánea de } \\
\text { evaluación del embarazo } \\
\text { ( } 15 \text { días después del primer }\end{array}$ & 52 & 54 & 326 & 595 & 176 & 115 а 268 \\
\hline $\begin{array}{l}\text { Consulta extemporánea de } \\
\text { seguimiento del embarazo } \\
\text { (entre las semanas } 28 \text { y } 32 \text { ) }\end{array}$ & 44 & 42 & 334 & 607 & 1,90 & 1,19 a 3,04 \\
\hline Vivir sin marido o apoyo conyugal & 21 & 31 & 357 & 618 & 1,17 & 0,64 a 2,14 \\
\hline Baja escolaridad de la madre & 14 & 15 & 364 & 634 & 1,63 & 0,73 a 3,60 \\
\hline
\end{tabular}
Hospital Gineco-Obstétrico Provincial de Sancti Spíritus, Cuba, 1994

${ }^{\mathrm{a}} \mathrm{RP}=$ Razón de posibilidades.

$$
\begin{aligned}
E= & \text { población expuesta al factor } \\
& \text { de riesgo. } \\
R R= & \text { riesgo relativo. }
\end{aligned}
$$

Solo se calculó el RAP con respecto a los factores que dieron un resultado significativo en el análisis multivariado y para ello se utilizó la prevalencia de la exposición entre los controles, por considerarse poco frecuente el resultado estudiado (14).

\section{RESULTADOS}

Hubo 378 casos y 649 controles. En el análisis bruto (no ponderado por estratos) se identificaron como factores de riesgo significativos de BPN la hipertensión arterial, la ruptura prematura de membranas, el aumento de peso menor de $8 \mathrm{~kg}$ durante el embarazo, el antecedente de BPN, tres o más embarazos anteriores, haber tenido abortos, el intervalo intergenésico menor de 24 meses, el hábito de fumar durante el embarazo, un primer control tardío del embarazo y consultas extemporáneas de evaluación y seguimiento gestacional. No resultaron significativas la falta de pareja, la baja escolaridad de la madre, ni la edad menor de 17 años o mayor de 35 (cuadro 1).

Después de introducir cada una de las variables que habían mostrado significación estadística en el análisis bruto, en el modelo multivariado final se obtuvo una $\chi^{2}$ de 60,61 con 5 grados de libertad, con una $P=0,04$ (cuadro 2). Con respecto a los casos que teóricamente se podrían evitar si se eliminara el factor de riesgo (RAP), destacan el aumento de peso menor de $8 \mathrm{~kg}$ $(19,37 \%)$, el antecedente de abortos anteriores $(10,44 \%)$ y el intervalo intergenésico menor de 24 meses $(9,22 \%)$. Los otros factores de riesgo incluidos en el modelo $1 / \mathrm{M}$, la hipertensión arterial durante el embarazo y la consulta extemporánea de evaluación, no aportaron RAP altos por su baja prevalencia entre los controles, aunque tuvieron $\mathrm{RP}$ importantes (1,92 y 1,65, respectivamente) (cuadro 2). 
CUADRO 2. Modelo multifactorial de regresión logística dicotómica (modelo final: $\chi^{2}=60,6$, con 5 g.l.) para bajo peso al nacer, en 378 casos y 649 controles. Hospital Gineco-Obstétrico Provincial de Sancti Spíritus, Cuba, 1994

\begin{tabular}{|c|c|c|c|c|c|}
\hline Factor de riesgo & $\mathrm{RP}^{\mathrm{a}}$ & $\begin{array}{l}\text { Estadístico } \\
\text { de Wald }\end{array}$ & $\begin{array}{l}\text { Grados de } \\
\text { libertad }\end{array}$ & $P$ & RAPPb \\
\hline Antecedente de abortos anteriores & 1,31 & 3,91 & 1 & 0,04 & 10,44 \\
\hline $\begin{array}{l}\text { Hipertensión arterial durante el embarazo } \\
\text { Consulta extemporánea de evaluación }\end{array}$ & 1,92 & 5,09 & 1 & 0,04 & 3,42 \\
\hline del embarazo & 1,65 & 5,51 & 1 & 0,02 & 5,13 \\
\hline Intervalo intergenésico <24 meses & 1,54 & 4,46 & 1 & 0,03 & 9,22 \\
\hline $\begin{array}{l}\text { Aumento de peso }<8 \mathrm{~kg} \text { durante el } \\
\text { embarazo }\end{array}$ & 2,50 & 35,28 & 1 & 0,00 & 19,37 \\
\hline
\end{tabular}

${ }^{a}$ Razón de posibilidades ajustada.

${ }^{b}$ Riesgo atribuible poblacional porcentual.

\section{DISCUSIÓN}

La mayoría de los factores de riesgo de BPN identificados en nuestro estudio aparecen en la literatura revisada con mayor o menor preponderancia, según los criterios establecidos para definir las exposiciones.

En relación con el antecedente de abortos anteriores, inducidos o espontáneos, encontramos un riesgo similar al que informa Sipila (2) para 1966, aunque distinto del que informa para 1986. Mandelson (5) solo pudo documentar un riesgo cuando eran dos o más los abortos anteriores. Pese a que en nuestro estudio la RP fue solo de 1,31, este factor aportó un RAP considerable debido a su prevalencia entre los controles. En este sentido, subrayamos la importancia de insistir en la prevención de los abortos, que contribuiría a la prevención del BPN.

La hipertensión arterial durante el embarazo mostró en nuestro estudio un riesgo relativo ajustado cercano a 2, similar al detectado por Wessel (3) y Manganaro (6), que confirmó que el BPN en hijos de madres hipertensas se debía a un crecimiento intrauterino retardado. Pereira Solla (7) también identificó un riesgo relativo superior a 2, muy similar al detectado por nosotros y por Bortman (15), que lo caracterizó de preeclampsia o eclampsia.

En este estudio identificamos que las mujeres que tuvieron tanto un control tardío del embarazo como las evaluaciones subsiguientes fuera del tiempo estipulado, presentaron un riesgo 1,65 veces mayor de $\mathrm{BPN}$, lo que concuerda con otros hallazgos en mujeres con cuidados prenatales inadecuados $(8,9)$. En otro estudio (15), en donde la variable se define como la oportunidad de la primera consulta prenatal, se encontró un riesgo 2,18 veces superior en mujeres que la tuvieron durante el cuarto o quinto mes, y 2,93 superior en las que la tuvieron después del quinto mes.
Un intervalo intergenésico menor de 12 meses se ha identificado como factor de riesgo en algunas investigaciones $(7,13)$. A pesar de que en nuestro estudio se fijó este intervalo en menos de 24 meses, esta variable también resultó riesgosa. Estos resultados apoyan la importancia de planificar no solo el número de hijos, sino también el espaciamiento de los embarazos.

Estudios recientes realizados en Suecia (12) y Canadá (16) han identificado el poco aumento de peso durante el embarazo como factor de riesgo de BPN, lo cual coincide con nuestros hallazgos. Por su parte, Fuentes-Afflick et al. (17) lo identificaron como factor de confusión en poblaciones latinas. De las variables que integran la evaluación nutricional de la embarazada, el aumento de peso durante el embarazo es una de las más fáciles de medir en la atención primaria y una de las que pueden despertar sospecha de la existencia de otros factores, como la anemia y un pobre desarrollo fetal.

En nuestros resultados destacan el poco aumento de peso, el antecedente de abortos y el intervalo intergenésico corto como los elementos que deben vigilarse más en la atención primaria. De esta manera se podría prevenirlos y mantener los buenos indicadores que muestra la provincia de Sancti Spíritus en su programa maternoinfantil (18), que son comparables con los de muchos países desarrollados (19).

Finalmente, cabe preguntarnos: ¿cuánto lograremos mejorar nuestros indicadores en este programa si continuamos trabajando en la prevención primaria de los factores de riesgo aquí identificados?

\section{REFERENCIAS}

1. Abdulrazzaq YM. Obstetric risk factors affecting incidence of low birth weight in live-born infants. Biol Neonate 1995;67:160-166.

2. Sipila P, Hartikainen AL, Von Wendt L, Oja $\mathrm{H}$. Changes in risk factors for unfavorable pregnancy outcome among singletons over twenty years. Acta Obstet Gynecol Scand 1994;(73):612-618.

3. Wessel H, Cnattingius S, Bergstrom S, Dupret A, Reimaier P. Maternal risk factors for pre- term birth and low birthweight in Cape Verde. Acta Obstet Gynecol Scand 1996;75(4): 360-366.

4. Bratton SL, Shoultz DA, Williams MA. Recurrence risk of low birthweight deliveries among women with a prior very low birthweight delivery. Am J Perinatol 1996;13(3): 147-150.

5. Mandelson MT, Maden CB, Daling JR. Low birth weight in relation to multiple induced abortions. Am J Public Health 1992;82(3): 391-394.

6. Manganaro R, Mami C, Marando N, Paolata A, Palmara A, Gemelli M. Infants born to hypertensive mothers: a clinical epidemiological study. Minerva Ginecol 1996;48(3): 73-76.

7. Santos Pereira Solla JJ, Guimaráes Pereira RA Medina MG, Pinto LL. Análisis multifactorial de los factores de riesgo de bajo peso al nacer 
en Salvador, Bahía. Rev Panam Salud Publica 1997;2(1):1-6.

8. Bener A, Abdulrazzg YM, Dawodu A. Sociodemographic risk factors associated with low birthweight in United Arab Emirates. J Biosoc Sci 1996;28(3):339-346.

9. Brown HL, Watkins K, Hiett AK. The impact of the women, infant and children food supplement program on birth outcome. Am J Obstet Gynecol 1996;174(4):1279-1283.

10. Cobas JA, Balcazar H, Benin MB, Keith VM, Chong Y. Acculturation and low birth weight infants among women: a reanalysis of HHANES data with structural equation models. Am J Public Health 1996;86(3):394-396.

11. Spinillo A, Capuzzo E, Iasci A, Nicola S, Piazzi G, Baltaro F. Sociodemographic and clinical variables modifying the smoking-related risk of low birth weight. Int J Gynaecol Obstet 1995:51(1):15-23.
12. Otterblad Olausson PM, Cnattin Gius S, Goldenberg RL. Determinants of poor pregnancy outcomes among teenagers in Sweden. Obstet Gynecol 1997;89(3):451-457.

13. Fourn L, Goulet L, Seguin L. Birth intervals and birth of low weight infants in Benin. Med Trop (Mars) 1996;56(2):163-166.

14. Coughlin SS, Benichou J, Weed DL. Estimación del riesgo atribuible en los estudios de casos y controles. Bol Oficina Sanit Panam 1996;121(2):114.

15. Bortman M. Factores de riesgo de bajo peso al nacer. Rev Panam Salud Publica 1998;3(5): 314-321.

16. Muscati SK, Koski KG, Gray-Donal K. Increased energy intake in pregnant smokers does not prevent human fetal growth retardation. J Nutr 1996;126:2984-2989.

17. Fuentes-Afflick E, Hessol NA. Impact of Asian ethnicity and national origin on infant birth weight. Am J Epidemiol 1997;145: 148-155.

18. República de Cuba, Ministerio de Salud Pública, Dirección Nacional de Estadísticas. Anuario estadístico. República de Cuba: MSP; 1996.

19. Guyer B, Martin JA, Mac Dorman MF, Anderson RN, Strobino DM. Annual Summary of Vital Statistics, 1996. Pediatrics 1997;100(6): 905-918.

Manuscrito recibido el 26 de junio de 1998 y aceptado para publicación, tras revisión, el 16 de marzo de 1999

ABSTRACT The objective of this work was to identify some risk factors that women could present during pregnancy and that are associated with low birthweight (less than $2500 \mathrm{~g}$ ). A study was performed during 1994 with 378 cases and 649 controls at the Provincial Obstetric-Gynecologic Hospital of Sancti Spíritus, Cuba. The data were obtained from clinical histories, the registry of births, and personal interviews with mothers. A bivariate analysis was carried out and possible confounding factors were controlled utilizing dichotomous logistic regression, using the Epi Info 5 and SPSS software programs. With the final multivariate model, the following risk factors for low birthweight were identified as significant: hypertension during pregnancy, infrequent checkups during pregnancy, previous abortions, a period of less than 2 years since the last birth, and a maternal weight increase of less than $8 \mathrm{~kg}$. Priority should be given to these last three variables in order to maintain the high standards of the maternal and child health program of Sancti Spíritus Province, Cuba. 